\title{
The Galactic abundance gradient from Cepheids
}

\section{Transition zone between 10 and $11 \mathrm{kpc} \star, \star \star$}

\author{
S. M. Andrievsky ${ }^{1,2}$, R. E. Luck ${ }^{1}$, P. Martin ${ }^{\star \star \star, 3}$, and J. R. D. Lépine ${ }^{4}$ \\ 1 Department of Astronomy, Case Western Reserve University, 10900 Euclid Avenue, Cleveland, OH 44106-7215, USA \\ e-mail:[sergei; luck]@fafnir.astr.cwru.edu \\ 2 Department of Astronomy, Odessa State University, Shevchenko Park, 65014, Odessa, Ukraine \\ 3 Canada-France-Hawaii Telescope, PO Box 65-1238, Mamalahoa Hwy, HI 96743, USA \\ e-mail: martin@cfht . hawaii.edu \\ ${ }^{4}$ Instituto de Astronomia, Geofísica e Ciências Atmosféricas, Universidade de São Paulo, Cidade Universitária, São Paulo, SP, \\ Brazil \\ e-mail: jacques@astro.iag.usp.br
}

Received 20 June 2003 / Accepted 11 September 2003

\begin{abstract}
This paper reports on the spectroscopic investigation of 12 Cepheids which are situated in the crucial region of galactocentric distances from $9 \mathrm{kpc}$ to $12 \mathrm{kpc}$, where according to our previous results (Andrievsky et al. 2002c; Luck et al. 2003) the radial metallicity distribution experiences an obvious change. In particular, the wriggle in the iron abundance distribution is found to fall approximately at galactocentric distances $10-11 \mathrm{kpc}$ (assuming galactocentric distance of the Sun $R_{\mathrm{G}, \odot}=7.9 \mathrm{kpc}$ ). Within the transition zone from 10 to $11 \mathrm{kpc}$ the relative-to-solar iron abundance decreases approximately to -0.2 dex. The new sample of stars, analyzed in present paper, gives results supporting the previous conclusion about the multimodal character of the metallicity distribution in galactic disc. Using a quite simple consideration of galactic chemical evolution we show that the observed distribution can be explained in the framework of a model which includes the spiral arms. In particular, the wriggle feature associated with $R_{\mathrm{G}} \approx 11 \mathrm{kpc}$ can be interpreted as a change of metallicity level in the vicinity of the galactic corotation resonance.
\end{abstract}

Key words. stars: abundances - Galaxy: abundances - Galaxy: evolution - stars: variables: cepheids

\section{Introduction}

In two previous papers (Paper III and Paper IV) from this series (Andrievsky et al. 2002a, 2002b, 2002c - Papers I, II, III respectively; Luck et al. 2003 - Paper IV) we described the characteristic features of the metallicity distribution across galactic disc, as derived from Cepheid variable stars. In Papers III and IV we noted that there is an apparent step-like character in the iron (and also some other elements) abundance distribution, and the wriggle (or change in the slope) of the abundance distribution is associated with galactocentric distances around

Send offprint requests to: $\mathrm{S}$. M. Andrievsky,

e-mail: scan@deneb.odessa.ua

* Based on spectra collected at the 3.6-m Canada-France-Hawaii Telescope.

$\star \star$ Table A1 (Appendix) is only available in electronic form at the CDS via anonymous ftp to

cdsarc.u-strasbg.fr $(130.79 .128 .5)$ or via

http://cdsweb.u-strasbg.fr/cgi-bin/qcat?J/A+A/413/159

$\star \star \star$ Visiting astronomer, Canada-France-Hawaii Telescope, which is operated by the National Research Council of Canada, the Centre National de la Recherche Scientifique of France, and the University of Hawaii. approximately 10-11 kpc. Twarog et al. (1997) also found such a change (discontinuity) which is imprinted in the metallicity distribution from open clusters. A similar conclusion has been also reached by Caputo et al. (2001) for galactic Cepheids, although with a rather high uncertainty.

Any structure within the metallicity distribution, such as the detected step-like metallicity change, if it is proved to be real, may have some important consequences for scenarios of the formation and evolution of our Galaxy. However, before evaluating the impact of the structure in the metallicity distribution on the galactic models, it should be completely proven that a wriggle/slope change near $R_{\mathrm{G}} \approx 11 \mathrm{kpc}$ does really exist. For example, it is not impossible that such a feature could be an artificial result from a statistically limited sample of distant Cepheids, and thus may not be seen for a larger sample of stars. On the other hand, even being real, the slope change may turn out to be a purely local phenomenon, which results from a possible short-scale inhomogeneity in the disc, and thus seen in a restricted longitudal range. It should be noted that our sample of distant Cepheids examined in Papers III and IV is in fact biased on the basis of the galactic longitude, so that the great majority of investigated stars fall in the region of $l$ from $190^{\circ}$ to $250^{\circ}$ (it is interesting to note that all distant open clusters 
investigated by Twarog et al. fall in the same region: $l$ from $130^{\circ}$ to $260^{\circ}$ ). Nevertheless, even for a limited region of galactic longitudes, the existence of a discontinuity, and its exact position, has to be precisely established.

With this aim, we present the results of the abundance determination for 12 galactic Cepheids that have not been investigated spectroscopically before (at least for detailed elemental abundance determination). These stars have galactocentric distances placing them within the crucial region between $R_{\mathrm{G}} \approx 9 \mathrm{kpc}$ and $12 \mathrm{kpc}$, where the feature in the metallicity distribution is seen.

\section{Observations and data reduction}

The observations were carried out on 1-5 February 2003 with the 3.6-m Canada-France-Hawaii Telescope. The fiber-fed Gecko spectrograph equipped with EEV1 $2048 \times 4500$ CCD (binned $2 \times 2$ ) was used. The resolving power provided by this combination was about 50000 . For each program star six consecutive non-overlapping spectra were secured. They are centered at $6040 \AA$, $6130 \AA, 6220 \AA, 6310 \AA, 6400 \AA$ and $6490 \AA$, and the length of each fragment is about $80 \AA$.

As a rule, the exposure time for one spectrum was $30 \mathrm{~min}$. The estimated $S / N$ ratio at the continuum level depends upon the wavelength interval, and varies for each star in the range from about 70 to 150, with one exception (V335 Aur) where the $S / N$ is about 40 .

Table 1 contains some details concerning our program Cepheid observations, as well as some information about the physical properties of Cepheids themselves (see next section).

The spectra were extracted from the raw frames using standard IRAF procedures including correct scattered light subtraction. They were continuum normalized, wavelength calibrated, and had equivalent widths measured using the WINDOWS compatible DECH20 package (Galazutdinov 1992).

Since the Gecko spectrograph has not been used previously for this study, a comparison of independently measured equivalent widths in the spectra of program stars would be desirable. Because none of our Cepheids has been observed before at high resolution, such a comparison was performed for the wellstudied star $\mu$ Per which was included in the list of our program stars specifically for this aim. Our equivalent widths $(E W)$ for this star have been compared with those measured from the ELODIE spectrum having the resolving power 40000 (1.93-m telescope of Haute-Provence Observatoire, France). As seen in Fig. 1, the independent data are in excellent agreement.

\section{Atmospheric parameters, abundances, and distances}

In Paper I we gave a thorough description concerning the method of determination of the atmospheric parameters, elemental abundances, and estimating the galactocentric distances. The same methods were also used in Papers II-IV from this series. It should be noted that because each observed spectrum spans only $80 \AA$, and because for each of our program stars six spectra were secured successively (the total exposure time is about $3 \mathrm{~h}$ ), these spectra were combined into a single

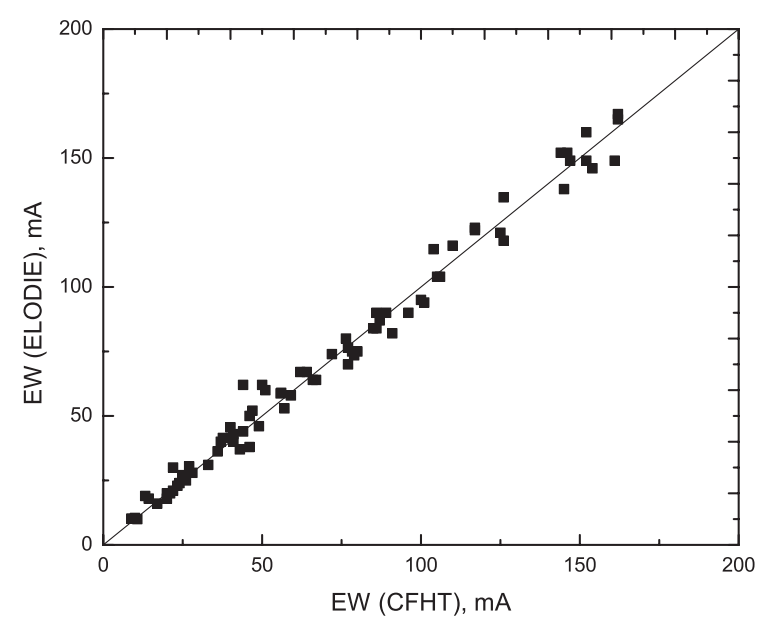

Fig. 1. Equivalent widths comparison for $\mu$ Per.

one, and that spectrum was then used for analysis. The atmospheric parameters for each star were determined at the pulsational phase which corresponds to the beginning of the fourth exposure. Since the total exposure time constitutes only about a few per cents of the pulsational period, we consider the derived parameters to be representative for the whole combined spectrum.

\subsection{Distances}

Specifically, for the determination of the Cepheid galactocentric distance the following formula was used:

$R_{\mathrm{G}}=\left[R_{\mathrm{G}, \odot}^{2}+(d \cos b)^{2}-2 R_{\mathrm{G}, \odot} d \cos b \cos l\right]^{1 / 2}$

where $R_{\mathrm{G}, \odot}-$ galactocentric distance of the Sun $(7.9 \mathrm{kpc}$, McNamara et al. 2000), $d$ - heliocentric distance of the Cepheid, $l$ and $b$ - galactic longitude and latitude respectively.

To obtain the heliocentric distances we use:

$d=10^{-0.2\left(M_{\mathrm{v}}-<V>-5+A_{\mathrm{v}}\right)}$

and (Laney \& Stobie 1993):

$A_{\mathrm{v}}=\left[3.07+0.28(B-V)_{0}+0.04 E(B-V)\right] E(B-V)$

where $M_{\mathrm{v}}$ is the absolute magnitude, $\langle V\rangle$ is the mean visual magnitude, $A_{\mathrm{v}}$ is the line of sight extinction.

To estimate the absolute magnitude we used "absolute magnitude - pulsational period" relation (Gieren et al. 1998). Other input data for Eqs. (1)-(3) such as galactic coordinates, pulsational periods, mean visual magnitudes, colors and $E(B-V)$ values were taken from Fernie et al. (1995). Galactocentric distances for program stars are listed in Table 4.

\subsection{Fundamental parameters and abundances}

Effective temperatures of program stars were determined with $T_{\text {eff }}$-line depth relations (Kovtyukh \& Gorlova 2000), and their gravities were found using the method developed by Kovtyukh \& Andrievsky (1999). 
Table 1. Program stars: details of the observations and atmospheric parameters.

\begin{tabular}{rcccccccc}
\hline \hline Star & $<V>$ & Date & JD 2 452 670+ & $P, \mathrm{~d}$ & $\phi$ & $T_{\text {eff }}, \mathrm{K}$ & $\log g$ & $V_{\mathrm{t}}, \mathrm{km} \mathrm{s}^{-1}$ \\
\hline V335 Aur & 12.46 & 1 & 1.7867 & 3.41325 & $0.622^{a}$ & 5750 & 2.20 & 3.5 \\
AD Gem & 9.86 & 1 & 1.9056 & 3.78798 & $0.818^{b}$ & 5784 & 2.15 & 4.3 \\
TW CMa & 9.56 & 1 & 1.9919 & 6.99507 & $0.059^{c}$ & 6142 & 1.80 & 3.9 \\
MM Per & 10.80 & 2 & 2.7947 & 4.11841 & $0.358^{a}$ & 5746 & 2.15 & 3.4 \\
VZ CMa & 9.38 & 2 & 2.9498 & 3.12623 & $0.777^{d}$ & 6542 & 2.50 & 3.6 \\
AO Aur & 10.86 & 3 & 3.7748 & 6.76301 & $0.707^{a}$ & 5459 & 1.70 & 3.6 \\
AA Gem & 9.72 & 3 & 3.8905 & 11.30233 & $0.625^{e}$ & 5126 & 1.40 & 4.4 \\
RY CMa & 8.11 & 3 & 3.9771 & 4.67825 & $0.897^{c}$ & 6096 & 2.10 & 4.3 \\
RZ Gem & 10.01 & 4 & 4.7804 & 5.52929 & $0.325^{b}$ & 5906 & 2.20 & 3.1 \\
DX Gem & 10.75 & 4 & 4.9235 & 3.13749 & $0.353^{d}$ & 6096 & 1.90 & 2.8 \\
BB Gem & 10.90 & 5 & 5.8050 & 2.30821 & $0.589^{f}$ & 6035 & 2.40 & 3.0 \\
UY Mon & 9.39 & 5 & 5.9428 & 2.39797 & $0.674^{d}$ & 6181 & 2.20 & 2.7 \\
$\mu$ Per & 4.18 & 2 & 2.8904 & - & - & 5331 & 1.50 & 3.2 \\
\hline
\end{tabular}

Date - February 2003.

JD and $\phi$ are given for the beginning of the fourth spectrum exposure.

Phases are calculated with elements from: ${ }^{a}$ Schmidt et al. (1995), ${ }^{b}$ Szabados (1991), ${ }^{c}$ Vinko (1991), ${ }^{d}$ Berdnikov et al. (1997), ${ }^{e}$ Moffet $\&$ Barnes (1985), ${ }^{f}$ Bersier et al. (1994).

In the Appendix (Table A1) abundances for the stars of the present sample derived from lines of different ions are given along with statistical information about the abundances. Averaged abundances are given in Tables 2 and 3. As usual, the reference (i.e. solar) abundances are adopted following Grevesse et al. (1996).

It should be noted that abundances of such elements as C, $\mathrm{O}, \mathrm{Na}, \mathrm{Co}$ and heavy species are found from a very limited number of lines, mainly because of the limited observed spectral region. Nevertheless, we consider those abundances to be rather reliable, because the lines used in present study had been also used by us in our previous studies (Papers I-IV), and they did not display any anomalous behaviour compared to other lines of the considered elements.

The position of all Cepheids investigated by us (Papers IIV, and present study) with respect to the Sun is shown in Fig. 2. Note that together with the present sample of program stars, the total number of Cepheids, that have been used in our galactic abundance gradient studies, is 122 stars. The tendency of the metallicity to decrease towards the larger distances in the direction of galactic anti-center is obvious, although in this region a few stars with rather high metallicity can also be found among the low-metallicity Cepheids. Figure 2 shows that our sample of investigated Cepheids is biased. More observations are needed in order to cover the galactic field in other directions, and thus to investigate the longitudal behaviour of the metallicity in galactic disc.

\section{Abundance distributions}

Based on Tables 2-4, and results of Papers I-IV we constructed the radial distribution of the elemental abundances. Figure 3 presents such a distribution for iron, and Figs. 4-6 for other elements.

One should note a remarkable carbon overabundance found in a metal-deficient Cepheid AA Gem (Fig. 4).

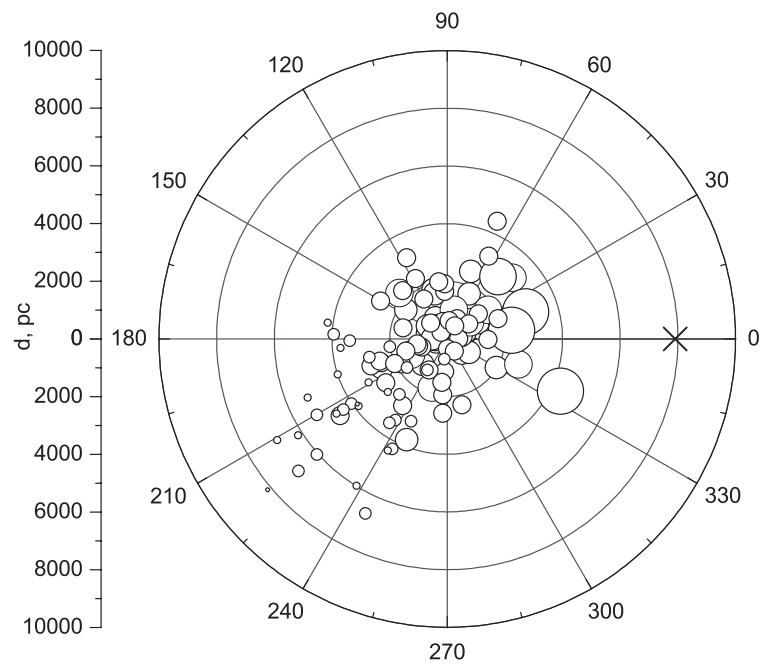

Fig. 2. Position of all investigated to date Cepheids (Paper I-IV, and present study) with respect to the Sun (in the origin). The sizes of circles represent the derived individual iron abundance: the larger size, the higher metallicity. Galactic center position is indicated by the cross.

The atmospheric ratio $[\mathrm{C} / \mathrm{Fe}]=+0.41$ dex for this star apparently exceeds that of SV Vul, for which Luck et al. (2001) have found $[\mathrm{C} / \mathrm{Fe}] \approx 0 \mathrm{dex}$, and on this base classified SV Vul as a Cepheid that is crossing the instability strip for the first time. A solar abundance of carbon is unusual for Cepheids, and the present case deserves a special separate study with a larger number of carbon lines.

Those elements in our program stars whose abundances are based on a significant number of lines $(\mathrm{Si}, \mathrm{Ni})$ do not result in additional increase of the scatter, thus the final abundance distributions of these elements (shown in Figs. 3-6) remain rather tight.

In this study we did not derive the abundances of $\mathrm{Mg}, \mathrm{Al}$, $\mathrm{Cu}, \mathrm{Zn}, \mathrm{Y}$ and $\mathrm{Gd}$ (as it was done in our previous papers) 
Table 2. Averaged relative-to-solar elemental abundances for program Cepheids: C-Mn.

\begin{tabular}{|c|c|c|c|c|c|c|c|c|c|c|c|}
\hline Star & $\bar{C}$ & $\mathrm{O}$ & $\mathrm{Na}$ & $\mathrm{Si}$ & $\mathrm{S}$ & $\mathrm{Ca}$ & $\mathrm{Sc}$ & $\mathrm{Ti}$ & $\bar{V}$ & $\mathrm{Cr}$ & $\mathrm{Mn}$ \\
\hline V335 Aur & - & - & -0.15 & -0.27 & - & - & - & - & - & - & -0.34 \\
\hline AD Gem & - & -0.36 & 0.07 & -0.24 & -0.42 & -0.17 & -0.06 & -0.08 & 0.11 & -0.09 & -0.25 \\
\hline TW Cam & -0.68 & -0.16 & -0.03 & -0.10 & -0.38 & -0.19 & -0.25 & 0.14 & 0.10 & -0.11 & -0.23 \\
\hline MM Per & -0.26 & -0.08 & 0.07 & -0.07 & -0.04 & -0.05 & -0.09 & 0.00 & -0.17 & -0.08 & -0.10 \\
\hline VZ CMa & -0.34 & -0.39 & 0.16 & 0.00 & -0.27 & -0.06 & -0.14 & 0.03 & 0.22 & 0.01 & -0.06 \\
\hline AO Aur & - & - & -0.03 & -0.24 & -0.51 & -0.35 & -0.34 & -0.04 & -0.16 & -0.25 & -0.28 \\
\hline AA Gem & 0.17 & 0.07 & 0.10 & -0.22 & -0.28 & -0.21 & -0.15 & -0.08 & -0.31 & -0.44 & -0.38 \\
\hline RY CMA & -0.27 & -0.30 & 0.16 & 0.04 & -0.23 & -0.02 & -0.14 & 0.16 & 0.15 & 0.08 & 0.04 \\
\hline RZ Gem & -0.32 & -0.23 & -0.06 & -0.13 & -0.33 & -0.27 & -0.13 & -0.02 & -0.12 & 0.00 & -0.22 \\
\hline DX Gem & -0.42 & -0.28 & 0.17 & 0.01 & -0.19 & -0.05 & -0.03 & 0.02 & -0.03 & 0.10 & -0.11 \\
\hline BB Gem & -0.31 & -0.45 & 0.13 & -0.07 & -0.37 & -0.31 & -0.10 & 0.24 & 0.12 & 0.06 & -0.20 \\
\hline UY Mon & -0.28 & -0.24 & 0.13 & -0.08 & -0.22 & -0.07 & -0.04 & 0.19 & 0.09 & 0.01 & -0.19 \\
\hline$\mu$ Per & -0.11 & -0.21 & 0.18 & 0.01 & -0.13 & -0.02 & -0.15 & 0.06 & -0.03 & -0.21 & -0.02 \\
\hline
\end{tabular}

Table 3. Same as Table 2 but for $\mathrm{Fe}-\mathrm{Eu}$.

\begin{tabular}{lrrrrrrrr}
\hline \hline Star & $\mathrm{Fe}$ & $\mathrm{Co}$ & $\mathrm{Ni}$ & $\mathrm{Zr}$ & $\mathrm{La}$ & $\mathrm{Ce}$ & $\mathrm{Nd}$ & $\mathrm{Eu}$ \\
\hline V335 Aur & -0.27 & - & 0.05 & - & - & - & - & - \\
AD Gem & -0.19 & - & -0.18 & - & 0.05 & -0.11 & 0.22 & 0.04 \\
TW Cam & -0.18 & - & -0.05 & -0.10 & 0.28 & -0.04 & 0.44 & -0.15 \\
MM Per & -0.01 & -0.03 & -0.15 & -0.16 & 0.18 & 0.06 & 0.44 & -0.08 \\
VZ CMa & -0.06 & - & -0.02 & -0.18 & 0.33 & -0.02 & 0.41 & 0.22 \\
AO Aur & -0.14 & 0.06 & -0.19 & - & 0.16 & 0.00 & 0.29 & -0.19 \\
AA Gem & -0.24 & -0.35 & -0.11 & 0.03 & 0.37 & -0.05 & 0.45 & 0.16 \\
RY CMA & 0.02 & 0.12 & 0.02 & -0.09 & 0.20 & -0.31 & 0.34 & -0.08 \\
RZ Gem & -0.12 & -0.06 & -0.07 & -0.29 & 0.32 & 0.01 & 0.36 & 0.00 \\
DX Gem & -0.02 & - & -0.11 & -0.12 & 0.41 & 0.01 & 0.42 & 0.04 \\
BB Gem & -0.09 & 0.08 & -0.09 & -0.12 & 0.22 & 0.31 & 0.12 & 0.14 \\
UY Mon & -0.08 & 0.29 & -0.03 & -0.05 & 0.32 & -0.27 & 0.42 & -0.06 \\
$\mu$ Per & -0.01 & -0.19 & 0.08 & -0.21 & 0.01 & -0.19 & 0.23 & -0.15 \\
\hline
\end{tabular}

Table 4. Physical and positional characteristics for program Cepheids.

\begin{tabular}{rrrccrc}
\hline \hline Star & $P, \mathrm{~d}$ & $d, \mathrm{pc}$ & $l$ & $b$ & $R_{\mathrm{G}}, \mathrm{kpc}$ & {$[\mathrm{Fe} / \mathrm{H}]$} \\
\hline V335 Aur & 3.41325 & 4180 & 172.22 & 3.98 & 12.04 & -0.27 \\
AD Gem & 3.78798 & 2772 & 193.27 & 7.63 & 10.59 & -0.19 \\
TW CMa & 6.99507 & 2549 & 229.12 & 0.12 & 9.76 & -0.18 \\
MM Per & 4.11841 & 2664 & 150.41 & -5.29 & 10.29 & -0.01 \\
VZ CMa & 3.12623 & 1252 & 239.86 & -4.44 & 8.59 & -0.06 \\
AO Aur & 6.76301 & 3939 & 177.61 & 1.99 & 11.83 & -0.14 \\
AA Gem & 11.30233 & 3709 & 184.59 & 2.70 & 11.60 & -0.24 \\
RY CMa & 4.67825 & 1233 & 226.01 & 0.28 & 8.80 & +0.02 \\
RZ Gem & 5.52929 & 2016 & 187.72 & -0.09 & 9.90 & -0.12 \\
DX Gem & 3.13749 & 2467 & 198.07 & 2.76 & 10.27 & -0.02 \\
BB Gem & 2.30821 & 2808 & 199.40 & 2.26 & 10.59 & -0.09 \\
UY Mon & 2.39797 & 2016 & 205.17 & 6.00 & 9.75 & -0.08 \\
\hline
\end{tabular}

because of the lack of available lines of these elements in the observed spectral region.

\section{Discussion}

The existence of the change in the metallicity distribution around 10-11 kpc was reported in Paper III, and statistically grounded for many elements in Paper IV. Our new abundances from the twelve new Cepheids located in the crucial region, outside the solar galactic orbit, suggest that a wriggle in the metallicity distribution at $R_{\mathrm{G}} \approx 10-11 \mathrm{kpc}$ does really exist. If we arbitrarily divide the whole range studied onto three parts (zone I: 4.0-6.6 kpc, zone II: 6.6-10.6 kpc, and zone III: 10.6$14.6 \mathrm{kpc}$, EE Mon is excluded), then the statistics for each zone are the following (Fig. 3 for the iron - our most reliable distribution):

Zone I

$[\mathrm{Fe} / \mathrm{H}]=-0.128( \pm 0.029) \times R_{\mathrm{G}}+0.930( \pm 0.170)$

$<[\mathrm{Fe} / \mathrm{H}]>=+0.174$ (s.d. $=0.129$, s.e. $=0.022$ ) 


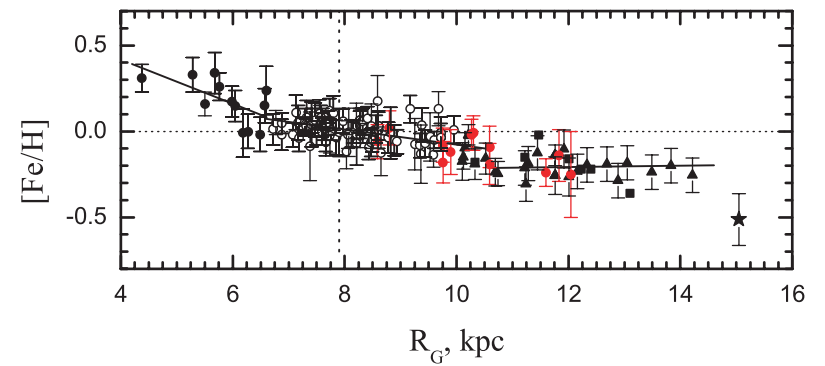

Fig. 3. The radial distribution of the iron abundance. Open circles the data from Paper I (the positions of some stars were corrected taking into account the results on abundances from Paper IV), black circles - the data from Paper II, black triangles and black squares - results from Paper IV combined with data from Paper III. Results from present study are shown by the red filled circles. $2 \sigma$ interval (standard deviation) is plotted for each star except for a few stars denoted as black squares (see Paper IV for explanation). The position of EE Mon is indicated by filled asterisk. Linear fits (solid lines) are shown for three zones (see discussion in the text).
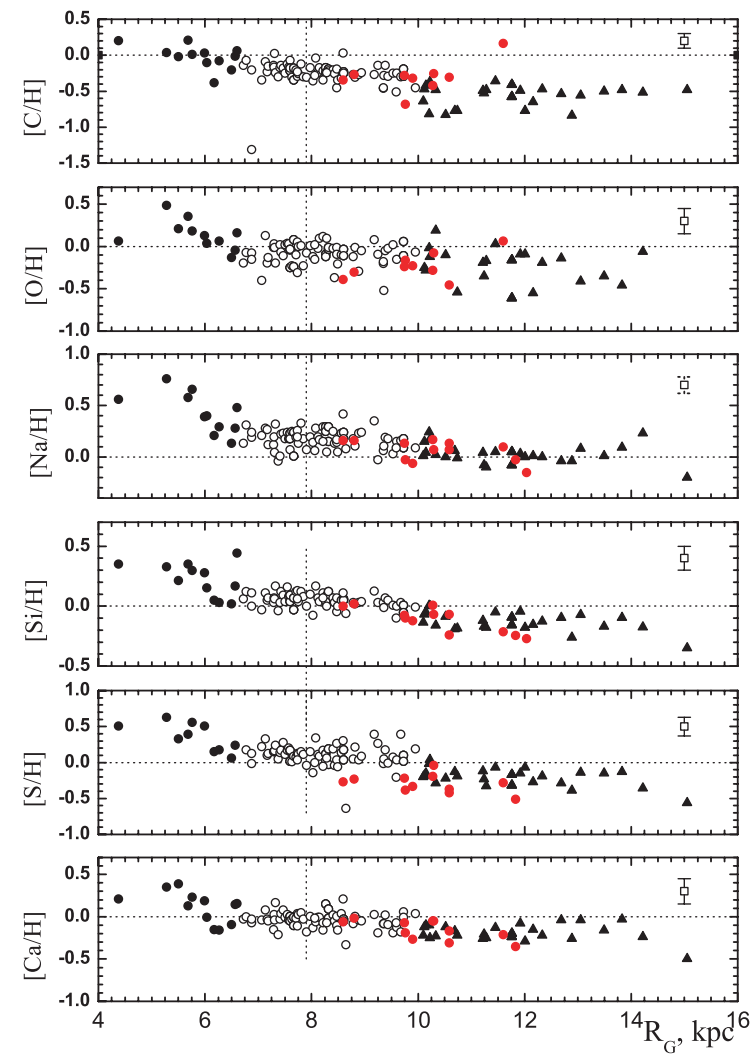

Fig. 4. Same as Fig. 3, but for elements C-Ca.The typical individual $2 \sigma$ interval is indicated.

$$
\begin{aligned}
& \text { Zone II } \\
& {[\mathrm{Fe} / \mathrm{H}]=-0.044( \pm 0.004) \times R_{\mathrm{G}}+0.363( \pm 0.032)} \\
& <[\mathrm{Fe} / \mathrm{H}]>=-0.012(\text { s.d. }=0.078, \text { s.e. }=0.005) \\
& \text { Zone III } \\
& {[\mathrm{Fe} / \mathrm{H}]=+0.004( \pm 0.011) \times R_{\mathrm{G}}+0.256( \pm 0.130)} \\
& <[\mathrm{Fe} / \mathrm{H}]>=-0.209(\text { s.d. }=0.058, \text { s.e. }=0.008) .
\end{aligned}
$$

As one can see, the mean metallicities for these zones are different. The difference in the slopes and mean metallicities across the disc is traced in the plots for e.g. Si, S, Ca, Ti, Cr, Mn,
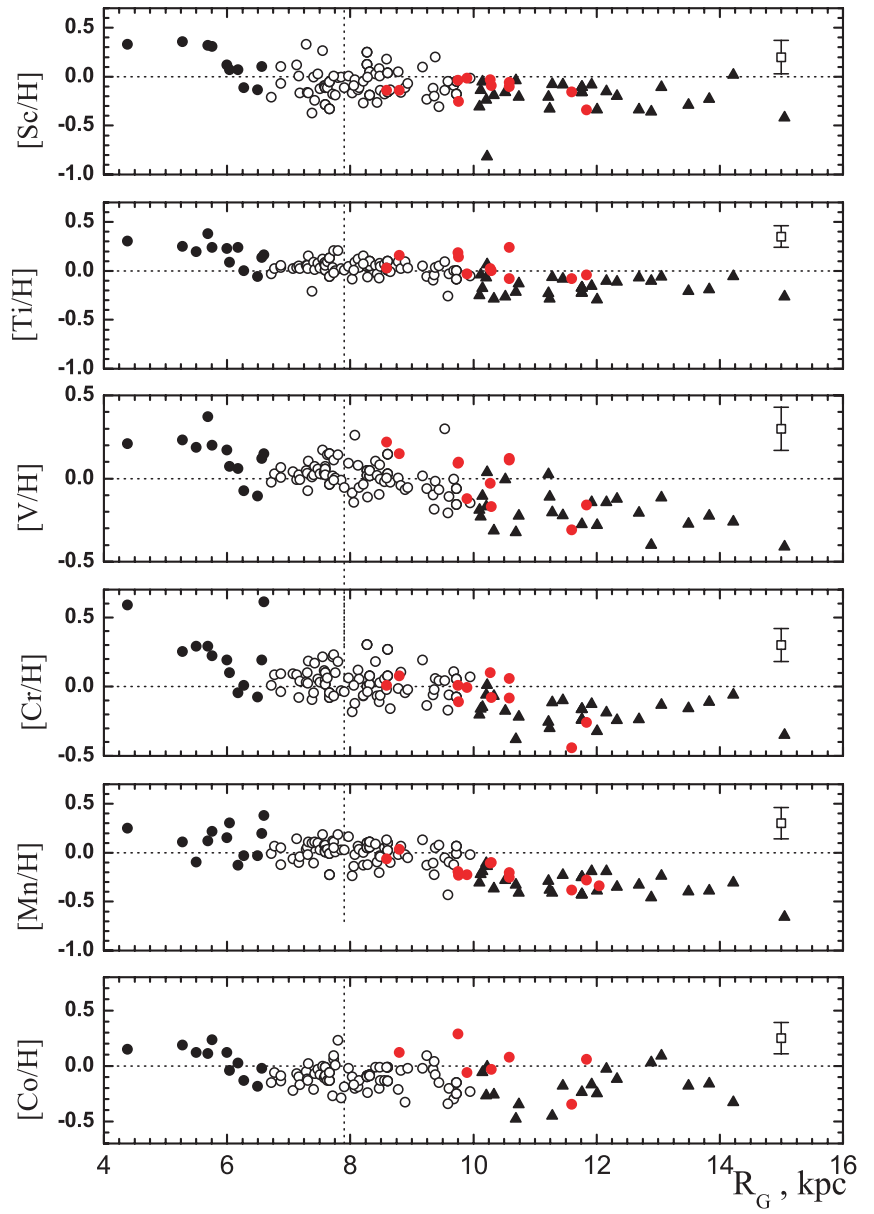

Fig. 5. Same as Fig. 3, but for elements Sc-Co.

Ni (Figs. 4-6), and also supported by Fig. 7 which shows the smoothed data on iron abundance distribution. The smoothing is performed using the locally weighted polynomial regression (LOWESS) method.

The paucity of stars in Figs. 3-7 at a galactocentric radius of about $11 \mathrm{kpc}$ is not due to a failure in the study to determine abundances for such stars but due to a lack of such stars as is demonstrated by Fig. 8 where we plot the galactocentric radius of 425 classical Cepheids from the Fernie et al. (1995) catalogue with known parameters of $(B-V), E(B-V)$, etc. The distances for these stars were calculated in the consistent way used in Papers I-IV, and also in the present paper. There is a clear gap at a radius of about $11 \mathrm{kpc}$. Why such a gap should exist is perhaps related to the corotation resonance (see Discussion).

What could be the reason for the complicated character of the metallicity distribution, and how significant is the detected structure of the metallicity distribution for enlarging our knowledge about the galactic disc properties? Below we give a simple consideration of this problem in the framework of galactic chemical evolution that may help to answer these questions. 

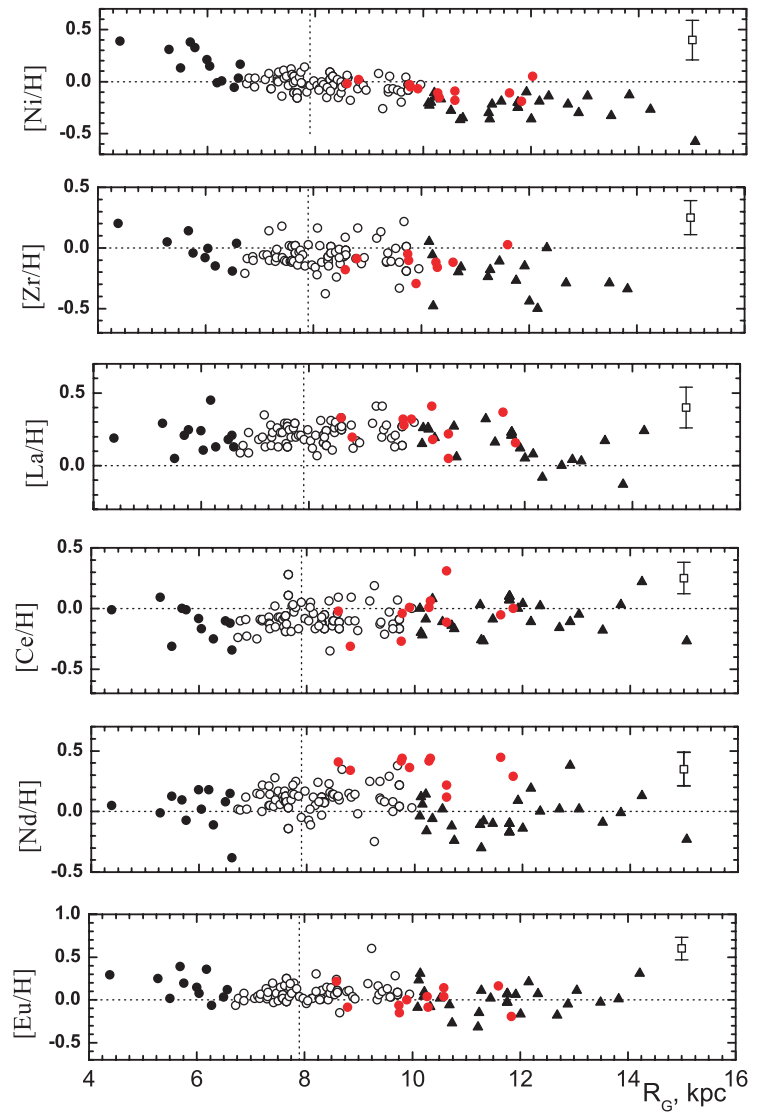

Fig. 6. Same as Fig. 3, but for elements Ni-Eu.

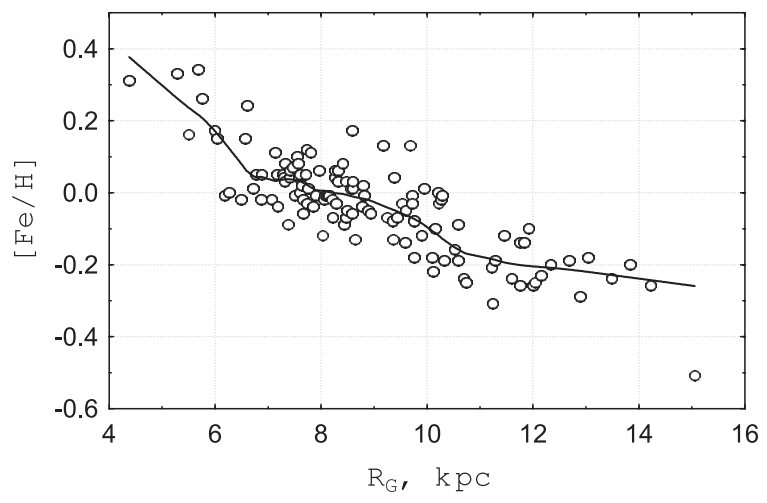

Fig. 7. Smoothed iron abundance distribution.

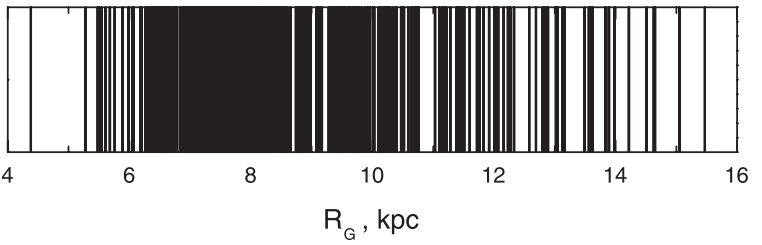

Fig. 8. The distribution of 425 galactic Cepheids on galactocentric radius. Each star is represented by a thin black vertical line.

\subsection{Recent results from the galactic models of chemical evolution}

The bibliography of papers devoted to the elaboration of galactic chemodynamical models based on different assumptions and approximations is quite numerous. It is beyond the scope of this paper to give a thorough description of all the models that have been presented. Nevertheless, it is worthwile to mention a few recent papers where the principal points of the theoretical/observational basis of such models can be found.

Boissier \& Prantzos (1999) considered the galactic chemical evolution with the star formation rate in a Schmidt-type form, which in addition includes a factor that depends upon galactocentric distance. They assumed that the infall rate is radially dependent (gradually increasing towards larger galactocentric radii). The authors succeeded in fairly well reproducing a number of the observational characteristics of the galactic disc. Further application of this model was described in Hou et al. (2001).

Chang et al. (2002) used a star formation rate that is determined by the energy balance between energy injection from $\mathrm{SNe}$ stars and energy dissipation in gas cloud collisions. Two gas infall rates were used - an exponential and a Gaussian form. As these authors pointed out, the chemical evolution and the resulting abundance gradients are different in the inner and outer parts of the Galaxy.

In a series of papers, Portinari \& Chiosi $(1999,2000)$ analyzed radial abundance gradients and gas profiles. They considered various star formation laws (the power Schmidt law, the Oort law, but in a form which includes the factor $R_{\mathrm{G}}^{-1}$, similar to Boissier \& Prantzos). According to a very important note in the first paper, none of the star formation laws considered was able to reproduce the observed metallicity gradient across the whole galactic disc. In the second paper Portinari \& Chiosi incorporated radial gas flows in order to better reproduce the observed radial abundance distribution. Since the actual profile of the radial velocity of the gas flow for large distances is not known, some simple examples of the velocity profile were considered. In addition, the authors estimated the influence of a galactic bar on the chemical evolution of the inner disc. Results obtained with the model including radial gas flows appeared to be more promising. Earlier, Thon \& Meusinger (1998) presented a model of galactic disc evolution which includes as an essential ingredient gas flows triggered by viscosity in the disc.

Abundance gradients and the Milky Way formation history were considered in a detailed study by Chiappini et al. (2001). The model created by these authors includes two gas infall episodes from the halo, the star formation rate in a modified Schmidt form, and a radial dependence of the characteristic time of infall. With a reasonable set of input parameters, this model is able to reproduce quite well the observed radial oxygen abundance distribution. Recently, Matteucci (2003) summarized the basic assumptions used for models of chemical evolution, and described the most essential results obtained to present in this field.

Despite progress in the understanding of the evolutionary properties of our Galaxy, and the numerous and quite successful attempts to reproduce some of the observational characteristics of the galactic disc, some work in this direction still remains. None of the previous models addressed the discontinuity discussed in our previous sections. Our primary goal is to address this specific point by using a quite simple scheme to estimate the role of spiral-arm triggered star formation, and to 
demonstrate the capability of such a simple model to explain some of the characteristic features of the radial metallicity distribution.

\subsection{Model assumptions}

A metallicity distribution with different slopes and different mean values within the different intervals of galactocentric distances could reflect some specific conditions in the disc, and may be shaped due to the various dynamical phenomena. For instance, it was demonstrated by Lacey \& Fall (1985), who considered the influence of radial gas flows (with schematic velocity profiles) on disc chemical evolution. Under special assumptions they succeeded to obtain in the calculations a metallicity distribution with a rather complicated character. The case of discontinuities (break in the slope) in the radial abundance gradients derived from $\mathrm{H}$ II regions has been discussed in the context of bar corotation radius for a few galaxies (Martin \& Roy 1995; Roy 1996). Friedli et al. (1994) considered the influence of bars on chemical composition in disc galaxies, and gave an explanation of the observed breaks. Pilyugin (2003) gave a critical consideration of this problem, and claimed an artificial origin of the reported bends or discontinuities in the slopes. Nevertheless, one should note that the paper of Pilyugin (2003) presents an alternative point of view, and at present his conclusion cannot be treated as already proven fact. The author doubts the reality of the bends of the slopes which are derived from the spectra of H II regions using the traditional method. In fact, this conclusion (if true) may only indicate that the results based on this particular method could be unreliable, but strictly speaking, it does not mean that bends do not exist.

Among other reasons, the appearance of some peculiarities in the radial abundance distribution could be partially connected to spiral structure and resonance phenomena in the galactic disc. Spiral structure may influence the star formation rate across the disc, and thus affect the metallicity level at a given galactocentric distance, while the resonances may represent some kind of potential barrier in certain parts of the galactic disc preventing the galactic gas from flows in the radial direction, thus, preserving a difference in the mean abundances between the separated zones. As a result, some change of the slope of the metallicity distribution could be expected in the vicinity of resonances.

A galactic spiral structure model uses as input parameters $\Omega\left(R_{\mathrm{G}}\right)$ - the angular velocity of the differentially rotating disc, $\Omega_{\mathrm{p}}$ - the angular velocity of the spiral pattern, and $\kappa\left(R_{\mathrm{G}}\right)$ - the epicyclic frequency $\left(\kappa\left(R_{\mathrm{G}}\right)=\left[4 \Omega\left(R_{\mathrm{G}}\right)+R_{\mathrm{G}} \frac{\mathrm{d} \Omega\left(R_{\mathrm{G}}\right)^{2}}{\mathrm{~d} R_{\mathrm{G}}}\right]^{1 / 2}\right)$. With the number of spiral arms $m$, the resonances (ILR - inner Lindblad resonance, OLR - outer Lindblad resonance, and CR - corotation resonance) appear under the following conditions:

$\Omega_{\mathrm{p}}=\Omega\left(R_{\mathrm{G}}=R_{\mathrm{ILR}}\right)-\kappa\left(R_{\mathrm{G}}\right) / m$,

$\Omega_{\mathrm{p}}=\Omega\left(R_{\mathrm{G}}=R_{\mathrm{OLR}}\right)+\kappa\left(R_{\mathrm{G}}\right) / m$,

$\Omega_{\mathrm{p}}=\Omega\left(R_{\mathrm{G}}=R_{\mathrm{CR}}\right)$.

In Fig. 9 the behavior of the disc angular velocity, as well as its combinations with epicyclic frequency for two cases $m=4$ and

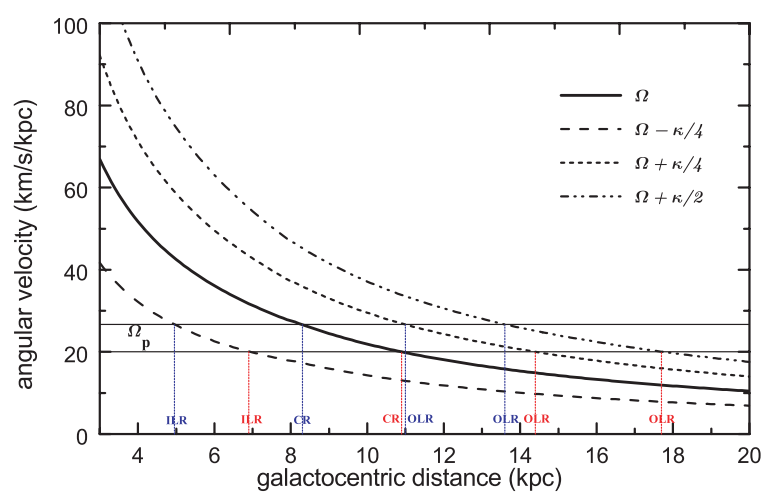

Fig. 9. Angular velocity vs. galactocentric distance, and the position of resonances. Two values of the pattern speed (20 and $27 \mathrm{~km} \mathrm{~s}^{-1} \mathrm{kpc}^{-1}$ ) are marked with two solid horizontal lines. The resonances attributed to the lower and higher value of the pattern speed are shown by red vertical lines and blue vertical lines respectively.

$m=2$ are shown (the diagram is based on the galactic rotation curve from Allen \& Santillan 1991).

The actual value of the pattern speed for our Galaxy is still debated in the literature. In the last decade it was realized that $\Omega_{\mathrm{p}}$ should be significantly higher than originally proposed by Lin et al. (1969) $\Omega_{\mathrm{p}}=11-13 \mathrm{~km} \mathrm{~s}^{-1} \mathrm{kpc}^{-1}$; but at the same time, there is at present no consensus about the exact value of this important parameter of the galactic model. For example, according to Marochnik et al. (1972), Creźé \& Mennessier (1973), Byl \& Ovenden (1978), Mishurov et al. (1979), Pavlovskaya \& Suchkov (1980) and Amaral \& Lépine (1997), the pattern speed in our Galaxy is in the range from 18 to $23 \mathrm{~km} \mathrm{~s}^{-1} \mathrm{kpc}^{-1}$. The most recently proposed value of the pattern speed (Pichardo et al. 2002; Bissantz et al. 2003) is $\Omega_{\mathrm{p}}=20 \mathrm{~km} \mathrm{~s}^{-1} \mathrm{kpc}^{-1}$ in line with the previous result. However, in some work a higher $\Omega_{\mathrm{p}}$ value has been found. For example, Avedisova (1989), Mishurov et al. (1997), Lépine et al. (2001), Fernandez et al. (2001) obtained a pattern speed in the range $26-30 \mathrm{~km} \mathrm{~s}^{-1} \mathrm{kpc}^{-1}$.

It is worth noting that Bissantz et al. (2003) emphazise that the real difference between their two theoretical models for $\Omega_{\mathrm{p}}=20$ and $40 \mathrm{~km} \mathrm{~s}^{-1} \mathrm{kpc}^{-1}$ "are too small to determine the spatial arm pattern speed reliably". Thus, the existing observational and theoretical methods do not provide enough accuracy to discriminate between the two possibilities of pattern speed value. Unfortunately, an accurate as possible $\Omega_{\mathrm{p}}$ value is necessary to determine the exact positions of the resonances in galactic disc.

With the lower value of the pattern speed $\left(\approx 20 \mathrm{~km} \mathrm{~s}^{-1} \mathrm{kpc}^{-1}\right)$, the resonances appear at the following galactocentric distances: $R_{\mathrm{CR}} \approx 10.9 \mathrm{kpc}, R_{\mathrm{ILR}} \approx 6.9 \mathrm{kpc}$ (four-armed disc), $R_{\mathrm{OLR}} \approx 14.4 \mathrm{kpc}$ (four-armed disc), $R_{\mathrm{OLR}} \approx 17.7 \mathrm{kpc}$ (two-armed disc). These values appear to be surprisingly close to those galactocentric distances that roughly separate individual regions on the smoothed curve of the metallicity distribution (Fig. 7). The first region has a larger slope, and it is separated from the second region at $R_{\mathrm{G}} \approx 6.5 \mathrm{kpc}$. The second and third regions both have the small slopes, and they are separated at $R_{\mathrm{G}} \approx 10-11 \mathrm{kpc}$. 
Outside of $15 \mathrm{kpc}$, the galactic disc is not sampled, but one of our program Cepheids gives a hint that the metallicity may drop further in this region.

With the higher value of the pattern speed the resonances are shifted towards smaller galactocentric distances. In this case, the wriggle at $10-11 \mathrm{kpc}$ may mark not the CR, as in the example considered above, but instead the OLR position. For this case to be valid, the required pattern speed should be close to $27 \mathrm{~km} \mathrm{~s}^{-1} \mathrm{kpc}^{-1}$ (see Fig. 9), which is exactly within the limits of estimates made by the authors claiming the higher value of $\Omega_{\mathrm{p}}$. The higher value ( $\Omega_{\mathrm{p}}$ is about $\left.27 \mathrm{~km} \mathrm{~s}^{-1} \mathrm{kpc}^{-1}\right)$, gives marking circles in the galactic disc with the following characteristics (see Fig. 9): $R_{\mathrm{CR}} \approx 8.3 \mathrm{kpc}, R_{\mathrm{ILR}} \approx 5.0 \mathrm{kpc}$ (four-armed disc), $R_{\mathrm{OLR}} \approx 11.0 \mathrm{kpc}$ (four-armed disc), $R_{\mathrm{OLR}} \approx$ $13.6 \mathrm{kpc}$ (two-armed spiral model).

Since the resonances may represent some kind of potential borders for the gaseous component of the disc, and the ILR and OLR may also limit the star formation rate, it is likely that the mean metallicities (and the slopes of metallicity distribution) should differ on either side of each border. To check this supposition, one should compare the calculated metallicity distribution, which is based on the galactic model with spiral arms, with our observational data showing some features associated with certain galactocentric distances. One can try to specify the actual value of the pattern speed using our abundance distribution data.

For a simple consideration of the galactic disc chemical evolution in conjunction with some of its dynamical characteristics, we have analyzed the following system of differential equations describing the time variations of the star and gas surface densities, and galactic gas metallicity:

$\frac{\mathrm{d} \mu_{\mathrm{s}}}{\mathrm{d} t}=(1-R) \psi$

$\frac{\mathrm{d} \mu_{\mathrm{g}}}{\mathrm{d} t}=-(1-R) \psi+f$

$\mu_{\mathrm{g}} \frac{\mathrm{d} Z / y}{\mathrm{~d} t}=(1-R) \psi-f Z / y$,

where $\mu_{\mathrm{s}}$ and $\mu_{\mathrm{g}}-$ are the surface density $\left(M_{\odot} \mathrm{pc}^{-2}\right)$ of the stars and gas respectively, $Z / y$ - the gas metallicity normalized to the yield of primary elements (following Maeder 1992 we denote " $y$ " as a net yield which is normalized to the mass confined in the stellar remnants), $\psi$ - the star formation rate $\left(M_{\odot} \mathrm{pc}^{-2} \mathrm{Gyr}^{-1}\right), R-$ the fraction of returned material to the ISM, $f$ - infall rate $\left(M_{\odot} \mathrm{pc}^{-2} \mathrm{Gyr}^{-1}\right)$. The metallicity of the infalling material was set to zero.

This system is similar to that described by Tinsley (1980). It has been analyzed in detail by Lacey \& Fall (1985), but with the space derivate terms on the right sides that depend upon the velocity of the gas radial flows (not considered in the present paper).

The basic equations representing the star formation and infall rates are the following:

$$
\begin{aligned}
& \psi=C \mu_{\mathrm{g}}^{k} \\
& f=\frac{\alpha^{2} M_{\mathrm{p}}}{2 \pi} \frac{\exp \left(-\alpha R_{\mathrm{G}}-t / \tau\right)}{\tau\left[1-\exp \left(-t_{\mathrm{p}} / \tau\right)\right]}
\end{aligned}
$$

Constant $C$ and power $k$ are specified below, $\alpha$ is an inverse characteristic distance for the infall rate in the disc $(1 / \alpha$ was adopted to be $4300 \mathrm{pc}$ ).

The latter Eq. (11) is the same as used by Lacey \& Fall (1985) with the present-day mass of the galactic disc $M_{\mathrm{p}}=6 \times$ $10^{10} M_{\odot}$, the age of Galaxy $t_{\mathrm{p}}=15 \mathrm{Gyr}$, and the characteristic time of infall $\tau$. In our calculations Eqs. (10) and (11) were modified, as described below.

Since it is commonly assumed that star formation may be partially triggered by gas deceleration in spiral arms, the star formation rate at a given galactocentric distance should therefore depend not only on the local surface gas density (Schmidt's law), but also on the difference of the angular velocities of the gas and spiral arms (Roberts 1970, 1972). The larger the difference, the higher the gas compression, and the corresponding star formation rate. It is quite likely that our Galaxy may be described by a combination four- and two-armed models, as proposed e.g. by Amaral \& Lépine (1997). To take these circumstances into account, we introduced instead of Eq. (10) the following expression:

$\psi=C \mu_{\mathrm{g}}^{k}(1+\Delta)$

and

$\Delta=\left(\epsilon_{1} \theta_{1}+\epsilon_{2} \theta_{1}\right) \frac{\left|\Omega\left(R_{\mathrm{G}}\right)-\Omega_{\mathrm{p}}\right| R_{\mathrm{G}}}{c_{\mathrm{s}}}$,

where $\epsilon_{1}$ and $\epsilon_{2}$ are optionally introduced factors which define an efficiency of the star formation in the 4- and 2-armed spirals respectively, the cut-off factors $\theta_{1}$ and $\theta_{2}$ are used following Mishurov et al. (2002), and $c_{\mathrm{s}}$ is the sound speed in the ISM (i.e. turbulent speed of the clouds). As pointed out by Englmaier \& Gerhard (1997) the sound speed in the ISM is of the order of 10-30 $\mathrm{km} \mathrm{s}^{-1}$, therefore we adopted an intermediate value of $20 \mathrm{~km} \mathrm{~s}^{-1}$.

In our calculations we used two different values for the efficiency parameter $\epsilon_{1}: 0.10$ and 0.20 and $\epsilon_{2}: 0.05$ and 0.10 (accordingly to Oort 1974, the efficiency of the star formation induced by the gas deceleration in spiral arms is small). It has been also assumed that both 4 - and 2-armed patterns rotate with the same angular velocity.

According to our definition, $\theta_{1,2}$ is equal to zero in the regions where the spiral pattern cannot exist (inside the ILR and outside the OLR circles). At the same time, between the resonance circles $\theta_{1,2}=1$ (see Fig. 9 for the position of resonances).

It should be noted that the star formation rate in the form given by Eqs. (12) and (13) has not been used before in the literature. The principal modification is that we propose to use a combined form for the star formation rate, while as a rule this input parameter has been used either in the Schmidt form, similar to Eq. (10), or in Oort's form, where it is assumed to be the monotonically decreasing function of the galactocentric distance $\sim R_{\mathrm{G}}^{-1}$ (see the recent review of Matteucci 2003). In contrast with a simple Schmidt's law, where the star formation rate depends only upon the surface gas density, or with a modified form used by Mishurov et al. (2002) in which the star formation is bound only to that part of the disc where the spiral pattern exists, and the star formation rate becomes negligible near to the corotation circle, our form allows star formation 
near the corotation circle, as well as at distances larger than the OLR position. (There exist obvious observational signs that the star formation process in our Galaxy is ongoing at rather large galactocentric distances, e.g. an existence of distant anti-center Cepheids.) Since near the CR the term $\Delta$ is small, the star formation in this specific region is well reproduced by the Schmidt power law. One has to note that a decreased star formation rate near the corotation resonance may result in the gap on the radial distribution of the Cepheids, which is actually seen in Fig. 8 around $11 \mathrm{kpc}$.

Several of our models were calculated with a variable factor $C$ in Eq. (12), which was assumed to slightly decrease linearly with galactocentric distance, attaining the numerical value of 0.18 at $R_{\mathrm{G}}=5 \mathrm{kpc}$ and 0.12 at $R_{\mathrm{G}}=11 \mathrm{kpc}$. In the solar neighborhood such a choice gives $C=0.15$. This, in our opinion, can simulate a dependence of the star formation rate upon the overall metallicity of the interstellar gas.

The spiral pattern may not be a permanently existing galactic structure, therefore in our calculations we arbitrarily adopted $\theta_{1,2}=0$ for all galactocentric distances during the time interval of the Galaxy evolution from $t=0$ to $6 \mathrm{Gyr}$ (the test calculations based on the supposition that the spiral pattern has been already formed at the epoch $t=1 \mathrm{Gyr}$ leave the main results practically unchanged).

Another important input parameter is the infall rate. As it was stated above, we adopted a basic equation describing the space and time dependence of the amount of infalling gas following Lacey \& Fall (1985). Similar to Hou et al. (2000), in some of our models we supposed that the characteristic time of infall is a function of the galactocentric distance (linear relation, which gives $\tau=3.5 \mathrm{Gyr}$ at the distance $4 \mathrm{kpc}$ and $6.5 \mathrm{Gyr}$ at $R_{\mathrm{G}}=8 \mathrm{kpc}$ ).

A set of input parameters for the models considered is given in Table 5 (this set is the same for models with $\Omega_{\mathrm{p}}=$ $20 \mathrm{~km} \mathrm{~s}^{-1} \mathrm{kpc}^{-1}$, models A20-L20, and $27 \mathrm{~km} \mathrm{~s}^{-1} \mathrm{kpc}^{-1}$, models A27-L27). The system of Eqs. (7)-(9) was solved with a 4 th-order Runge-Kutta integration for the time interval from $t=0$ to $15 \mathrm{Gyr}$. We have considered the range of galactocentric distances from $4 \mathrm{kpc}$ to $16 \mathrm{kpc}$. At distances smaller than $4 \mathrm{kpc}$ the disc chemical properties and evolution with time are most likely controlled by the central bar (see remarks made above), while beyond $16 \mathrm{kpc}$ the galactic disc properties are not well known.

\subsection{Model results}

The final results of the modeling are showed in Figs. 10-12 (models A20-L20), and in Figs. 13-15 (models A27-L27). Note that the model metallicity is normalized to the corresponding value at the galactocentric distance of the Sun $R_{\mathrm{G}, \odot}$. Such a choice can be criticized, of course, since doing it in this way we implicitly assume that the Sun's abundances (iron abundance, in particular) are representative of the presentday chemical composition of the ISM. Thus, by using normalized modeled metallicities $Z\left(R_{\mathrm{G}}\right) / Z\left(R_{\mathrm{G}, \odot}\right)$ for comparison with relative-to-solar abundances derived from Cepheids, we do not take into account that our Sun is 5 Gyr old, and might
Table 5. Input parameters for considered models.

\begin{tabular}{ccccccc}
\hline \hline Model & $k$ & $C$ & $\Delta$ & $\tau, \mathrm{Gyr}$ & $\epsilon_{1}$ & $\epsilon_{1}$ \\
\hline $\mathrm{A}$ & 1.0 & 0.2 & no & 6 & & \\
$\mathrm{~B}$ & 1.0 & 0.2 & yes & 6 & 0.10 & 0.05 \\
$\mathrm{C}$ & 1.0 & 0.2 & no & $\tau\left(R_{\mathrm{G}}\right)$ & & \\
$\mathrm{D}$ & 1.0 & 0.2 & yes & $\tau\left(R_{\mathrm{G}}\right)$ & 0.10 & 0.05 \\
\hline $\mathrm{E}$ & 1.4 & 0.1 & no & 6 & & \\
$\mathrm{~F}$ & 1.4 & 0.1 & yes & 6 & 0.10 & 0.05 \\
$\mathrm{G}$ & 1.4 & 0.1 & no & $\tau\left(R_{\mathrm{G}}\right)$ & & \\
$\mathrm{H}$ & 1.4 & 0.1 & yes & $\tau\left(R_{\mathrm{G}}\right)$ & 0.10 & 0.05 \\
\hline $\mathrm{I}$ & 1.0 & 0.2 & yes & 6 & 0.20 & 0.10 \\
$\mathrm{~J}$ & 1.0 & 0.2 & yes & $\tau\left(R_{\mathrm{G}}\right)$ & 0.20 & 0.10 \\
$\mathrm{~K}$ & 1.0 & $C\left(R_{\mathrm{G}}\right)$ & yes & 6 & 0.20 & 0.10 \\
$\mathrm{~L}$ & 1.0 & $C\left(R_{\mathrm{G}}\right)$ & yes & $\tau\left(R_{\mathrm{G}}\right)$ & 0.20 & 0.10 \\
\hline
\end{tabular}

Coefficient $C$ includes the factor $(1-R)$.

have a chemical composition different from that of the ISM in its neighborhood. Nevertheless, we should note that the mean metallicity value for the subsample of Cepheids in the direct vicinity of the Sun is practically solar, therefore such a comparison should be formally correct.

Our models A20 and A27 give the metallicity and surface densities of the stars and gas that are almost the same as produced by the similar model A of Lacey \& Fall (1985). As expected, in this case the model metallicity at each galactocentric distance reaches the same value (i.e. there is no metallicity gradient). It is demonstrated by the basic models A20 and A27 with a characteristic time-scale of infall $\tau=6 \mathrm{Gyr}$ and "switched-off" $\Delta$ term in the equation for the star formation rate. Models C20 and C27 produce a monotonically decreasing metallicity in the disc. Substantially different metallicity distributions are observed in models B20, B27, D20 and D27 where the term depending upon the relative velocity of the disc and spiral pattern contributes to the star formation rate. Models E20-H20 and E27-H27 give the corresponding results for the case of a stronger dependence of the star formation rate upon the surface gas density. Qualitatively the models with $k=$ 1.0 and 1.4 show similar radial distributions of $\mu_{\mathrm{s}}$ and $\mu_{\mathrm{g}}$. The set of models I20-L20 and I27-L27 were calculated with an increased efficiency of the star formation in the spiral arms, as well as in two cases with a variable factor $C$.

As one can see in Figs. 10-15, some models clearly show the wriggles and step-like features in the metallicity distribution in the vicinity of the resonances. We compared the theoretical metallicity distribution shown in Figs. 10-15 to the observational data (e.g. Fig. 3), and found that none of our models except for the model L20 (and perhaps L27) is able to satisfactory reproduce the observed metallicity distribution. As an example, in Figs. 16 and 17 we show the comparison between the metallicity produced by the models L20 and L27 and observations (iron abundance). Strictly speaking, the instantaneous recycling approximation (IRA) used by us implies a comparison with radial distributions of those elements which are produced mainly by massive stars (for example, oxygen and a few others). Unfortunately, the oxygen abundance derived from our program stars is much less reliable than, for example, the iron 

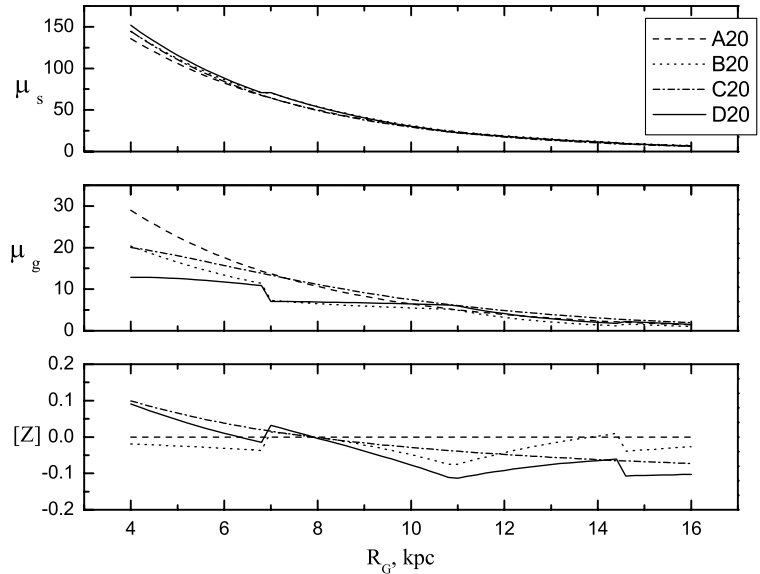

Fig. 10. The distribution of the star and gas surface densities, and metallicity in the disc at present epoch. Models A20-D20.
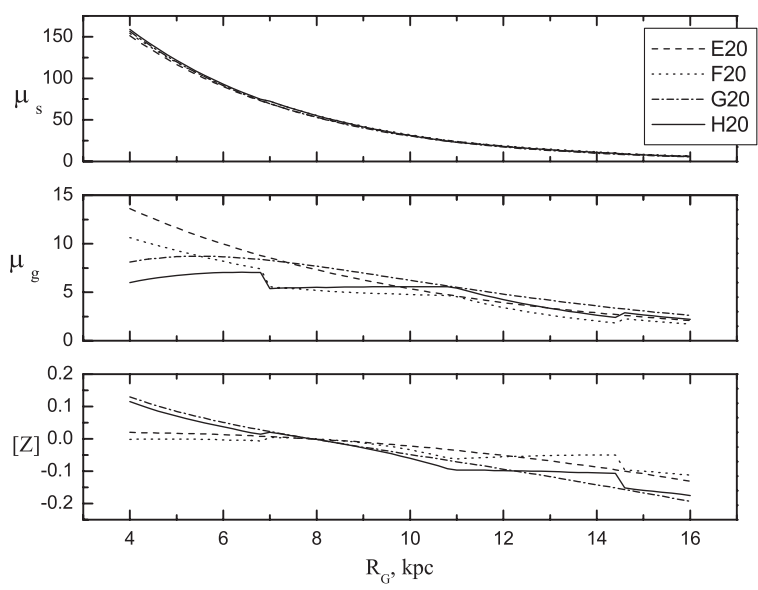

Fig. 11. Same as Fig. 10 but for models E20-H20.
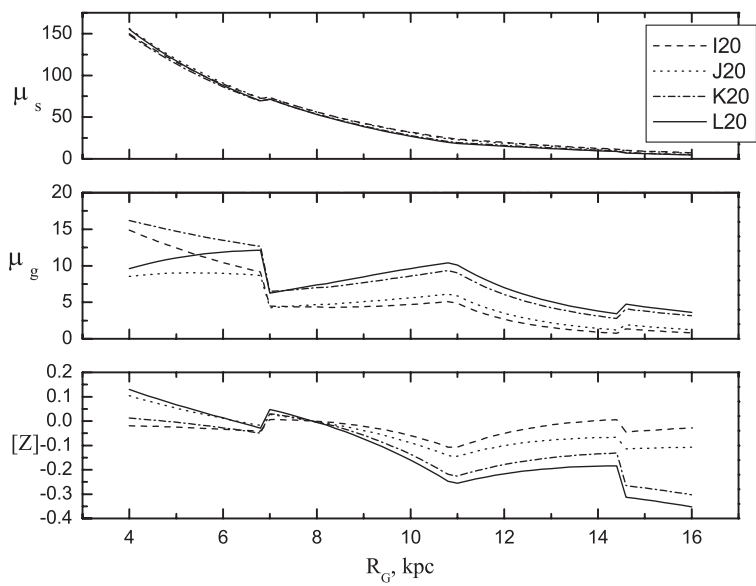

Fig. 12. Same as Fig. 10 but for models I20-L20.

abundance (only a few oxygen lines are analyzed for each star, while the number of iron lines varies from tens to hundreds for each star). We shall then assume, as a first approximation, that the global metallicity $Z$ can be compared with the measured iron abundance gradient, although models that do not assume an IRA would be certainly more adequate for such a comparison. The main features seen in the iron abundance
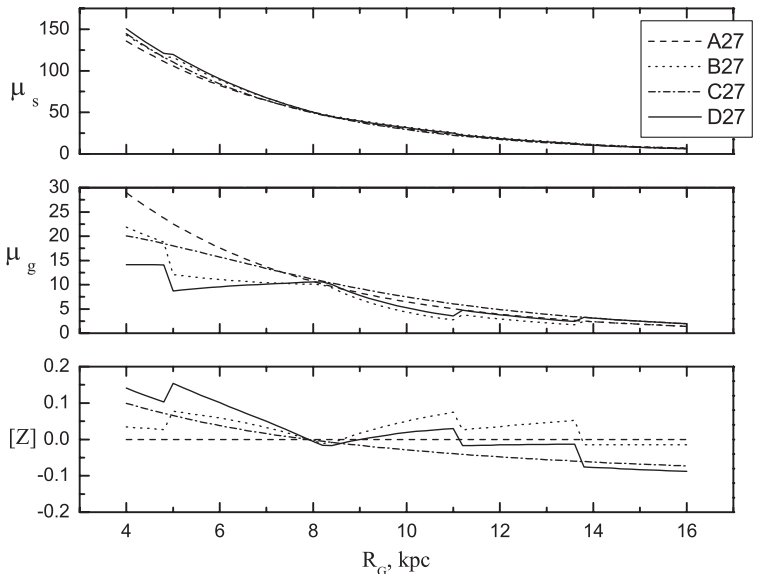

Fig. 13. Same as Fig. 10 but for models A27-D27.
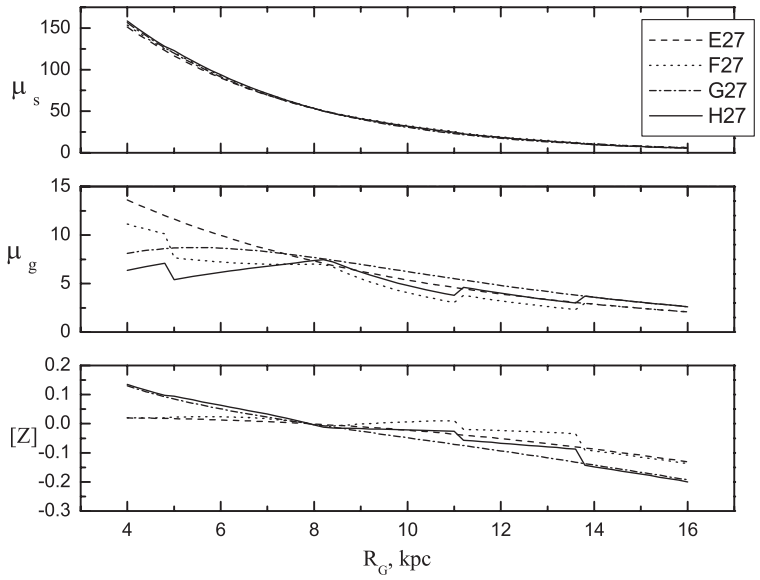

Fig. 14. Same as Fig. 10 but for models E27-H27.
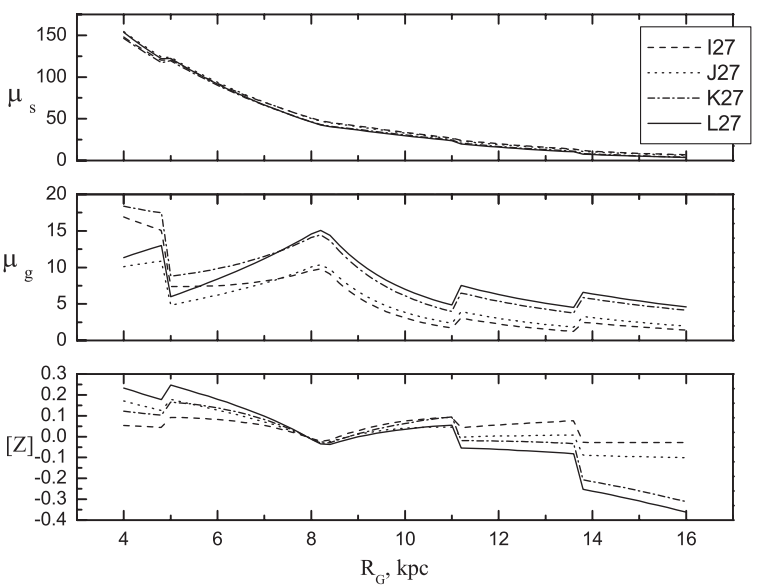

Fig. 15. Same as Fig. 10 but for models I27-L27.

distribution (Fig. 3) can also be traced for such elements as silicon, sulfur, calcium, titanium, chromium, manganese, nickel, which are also partially formed in SNe II explosions (Tsujimoto et al. 1995).

Although the agreement between the theoretical and observed data is only marginal for some galactocentric distances, generally the model "L" fairly well reproduces features of the actual metallicity distribution: the rather flat metallicity 


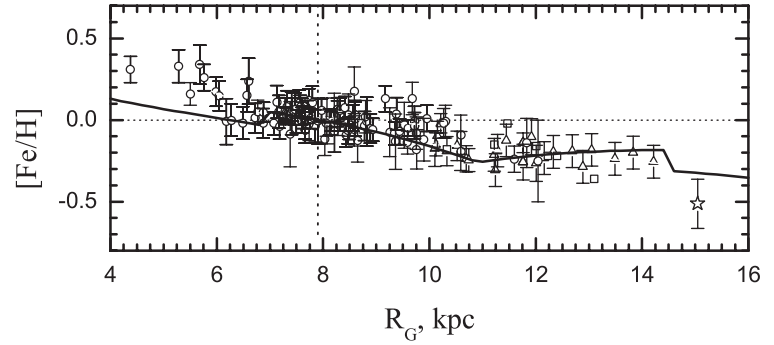

Fig. 16. The model metallicity distribution (model L20, thick solid line) superimposed on the observations from Fig. 3.

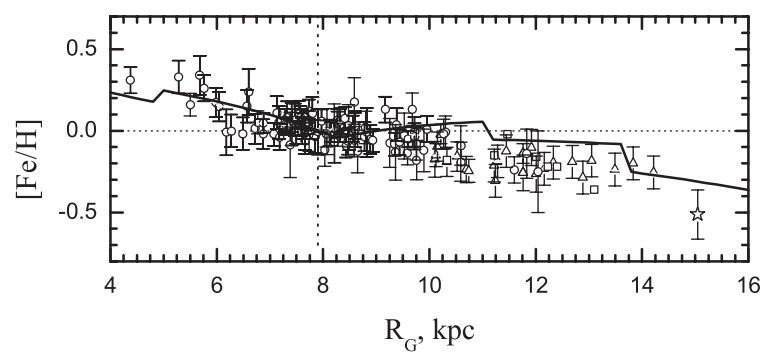

Fig. 17. Same as Fig. 16 but for model L27.

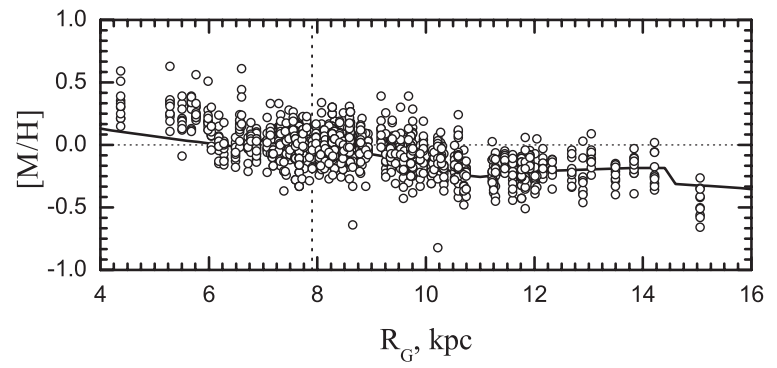

Fig. 18. Same as Fig. 16 but for other primary elements.

distribution in the middle part (distances from 7 to $9 \mathrm{kpc}$ ), the change of metallicity level and slope at approximately 10$11 \mathrm{kpc}$, and the apparently decreased metallicity in the outer part of the disc. Let us note again that in our model " $L$ " the star formation rate is proportional to the gas surface density, and, in addition, is enhanced due to the term which depends upon the relative velocity of the gas and spiral arms. This is combined with an infall rate which has a characteristic time-scale that progressively increases toward the larger radii. It is also important to emphasize that both successful models are based on the supposition that the coefficient in the star formation rate is a function of the galactocentric distance.

Qualitatively, the abundance distributions of such primary elements as $\mathrm{Si}, \mathrm{S}, \mathrm{Ca}, \mathrm{Sc}, \mathrm{Ti}, \mathrm{V}, \mathrm{Cr}, \mathrm{Mn}, \mathrm{Co}$ and Ni (Figs. 4-6) corroborate the general conclusion about the tendencies which can be traced in Figs. 16 and 17 for iron. It is demonstrated by Fig. 18 (primary elements + model L20) and Fig. 19 (the same + model L27) where the abundance distributions of these primary elements are plotted together, and then compared to the model predictions.

We can obtain even better agreement between the modeled metallicity distribution and the observed one (especially for the inner part of galactic disc) if we suppose that the characteristic time of an infall rate is an exponential function of the

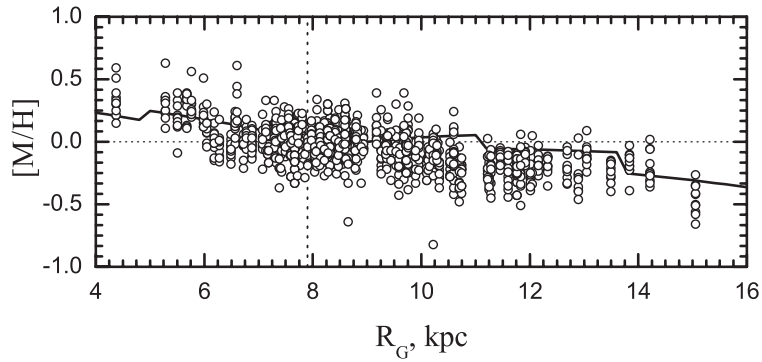

Fig. 19. Same as Fig. 18 but for model L27.
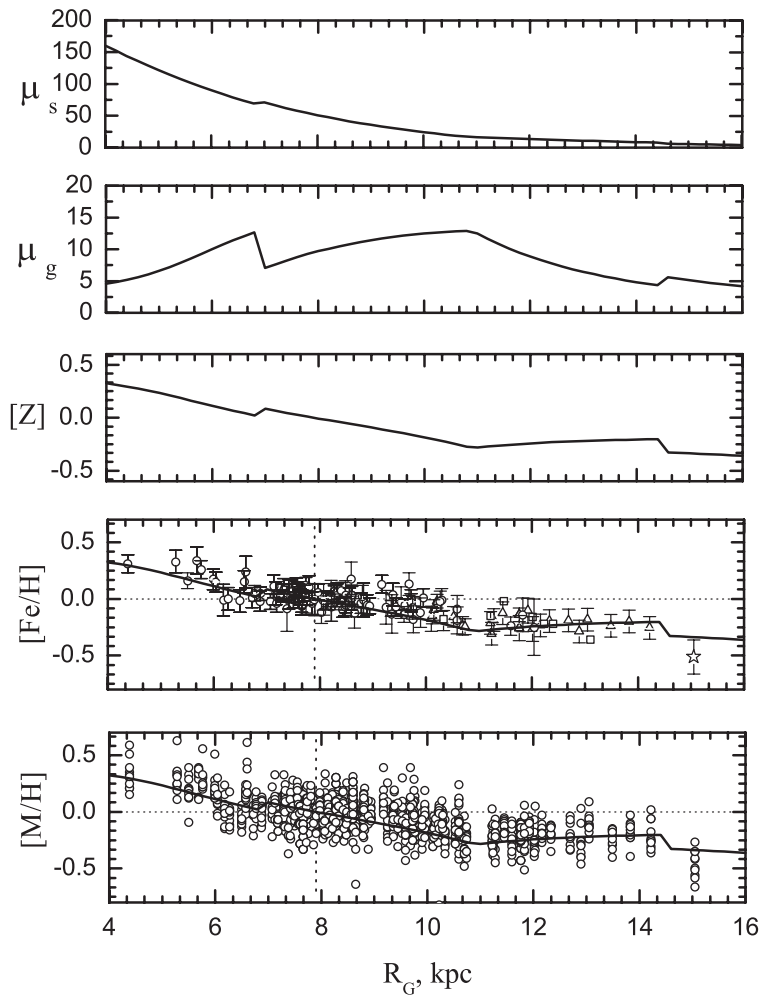

Fig. 20. Some results for the model L20 but with an exponential dependence of the infall rate upon the galactocentric distance.

galactocentric distance $\left(\tau\left(R_{\mathrm{G}}\right)=0.2 \exp \left(0.5 R_{\mathrm{G}} / 10^{3}\right) \mathrm{Gyr}\right)$. This is demonstrated in Fig. 20 where we plot results for model L20 modified with an exponential dependence of the infall rate upon the galactocentric distance. Despite a good agreement for the inner disc seen in Fig. 20, one should keep in mind that galactic bar is believed to play a key role in the chemistry of this region, but its influence on the chemical properties of the ISM was not considered in the present study.

We would like to note only a qualitative similarity between our modeled metallicity distribution and that derived from our program stars. We did not intend to go deeply into details of the disc chemical evolution, and with our simple models, we do not expect to achieve an exact fit to observed data. The main aim of this study was to further investigate the structure of the metallicity distribution in galactic disc, and to show the possibility of interpreting that structure by using basic characteristics of the spiral model of our Galaxy.

Even such quite simple models, like L20 (or L27), seem to display some promising results. According to these results 
the dynamical characteristics of the disc might play an important role in the formation of the chemical spatial peculiarities during galactic evolution, such as multimodal metallicity distributions with different slopes and mean metallicities within different disc zones. If we correctly understand the origin of these peculiarities, then they, in principal, can be used as a tool for evaluation of the star formation efficiency across the disc, determination of the approximate position of the Lindblad resonances, and thus they can provide an independent way to estimate the spiral pattern velocity. As previously noted, the latter is a very important, but poorly constrained parameter of the galactic chemodynamical model. Inspecting Figs. 16-20 one can conclude that $\Omega_{\mathrm{p}}=20 \mathrm{~km} \mathrm{~s}^{-1} \mathrm{kpc}^{-1}$ seems to be more the most preferable value for the Galaxy.

The change of the slope at $\sim 6-7 \mathrm{kpc}$ in the Milky Way may correspond in part to the position of the bar corotation circle (assuming that its radius is at $1.4 \times$ length of the bar, which seems to be a general "rule" in several barred galaxies). For example, Martin \& Roy (1995) found clear evidence of a break in the slope of the $\mathrm{O} / \mathrm{H}$ gradient near the bar corotation radius for the strongly barred galaxy NGC 3359 (a steep inner gradient, and very flat one outside the bar corotation). The existence of such a break can be understood in the framework of SPH simulation (Friedli et al. 1994) where the gas and stars are mixed on a large-scale by the bar, and changes in the slopes in the gaseous and stellar metallicity gradients are seen.

Additionally, the process of stellar galactic orbit diffusion can further change the metallicity distribution which is determined from a stellar population. For example, during its lifetime, a Cepheid can move a distance from its birthplace which is not negligibly small. If we adopt for the diffusion velocity a few $\mathrm{km} \mathrm{s}^{-1}$, and for the life-time about $10^{8} \mathrm{yr}$, then the travel distance can reach several hundred parsecs. Therefore, very sharp changes in the radial metallicity distribution, similar to those resulting from our simple simulation, can hardly be traced with a sample of Cepheid stars.

Finally, it should be noted that we do not consider the spiral arms a unique explanation of the non-linear behavior of the observed metallicity distribution. Moreover, as was shown above, to explain the rather significant increase of the metallicity level in the inner part of the disc, one needs to invoke some other assumption(s) - a galactic bar and associated gas flows, or a specific form of the infall rate radial dependence. It is also necessary to emphasize that other models (e.g. Portinari \& Chiosi 1999; Chiappini et al. 2001, among others) are also able to obtain gradients which are not linear, but show clear bendings of the metallicity distribution.

\subsection{Comparison with other observable characteristics}

Since when compared to the standard form of the star formation rate we have more than two free parameters (see Eq. (12)), it would be reasonable to use other observational constraints together with the abundance gradients in order to make an independent check of our basic suppositions. For example, the star and gas surface density distributions and/or their specific values in the solar neighborhood can be applied in such a check.
Table 6. Observed and computed parameters at $R_{\mathrm{G}, \odot}=7.9 \mathrm{kpc}$ and $t=15 \mathrm{Gyr}$.

\begin{tabular}{cccc}
\hline \hline Parameter & Model L20 & Model L27 & Reference \\
\hline$\mu_{\mathrm{g}}, M_{\odot} \mathrm{pc}^{-2}$ & 7.3 & 14.2 & $6-16(1,2)$ \\
$\mu_{\mathrm{s}}, M_{\odot} \mathrm{pc}^{-2}$ & 54.8 & 48.0 & \\
$\mu_{\mathrm{tot}}, M_{\odot} \mathrm{pc}^{-2}$ & 62.1 & 62.2 & $40-75(3)$ \\
$\mu_{\mathrm{g}} / \mu_{\mathrm{tot}}$ & 0.12 & 0.23 & \\
\hline
\end{tabular}

1 - Dickey (1993), 2 - Kulkarni \& Heiles (1987), 3 - Mera et al. (1998a,b).

We would like to emphasize at this point that we consider the abundance gradient as one of the most important and reliable observational input parameters. A common practice is to compare galactic model results with observational data on the combined molecular and atomic hydrogen density distribution, but it should be noted that observational distributions of $\mathrm{H}_{2}$ and $\mathrm{HI}$ are actually not purely observational parameters. For example, the $\mathrm{H}_{2}$ molecular gas surface density is obtained from $\mathrm{CO}$ emission surveys, and this result is dependent upon the assumed value of the galactic $\mathrm{CO}-$ to- $\mathrm{H}_{2}$ conversion factor. The different values of this factor available from the literature imply a rather significant uncertainty in the resulting value of the $\mathrm{H}_{2}$ surface mass density (Blitz 1996). This can lead to differences in absolute values of $\mathrm{H}_{2}$ surface mass density, see for instance, Blitz (1996) and Heyer et al. (2000); both papers are based on original observations from Dame (1993). There also exists another problem with $\mathrm{CO}$ emission data and related results on the molecular gas density. As was noted by Wong \& Blitz (2002), CO emission can be amplified simply due to the star formation process ongoing in molecular clouds, and in this case, $\mathrm{CO}$ emission may trace this process rather than the $\mathrm{H}_{2}$ mass. Thus, one can conclude that the molecular gas density based on a $\mathrm{CO}$ emission survey, in principal, can be overestimated due to this effect.

The situation with observational data on atomic hydrogen density is also rather complicated. The radial distribution of this parameter dramatically depends upon the adopted shape of the rotation curve (see a good example in Blitz 1996). According to Lockman (1988) and Dame (1993) even very small differences between the applied rotation curves can produce results of surface density that differ by $50 \%$. As an illustration of the above problem we refer the reader to the very recent investigations of the gas distribution in our Galaxy carried out by Olling \& Merrifield (1998) and Wolfire et al. (2003).

Nevertheless, despite these problems, we compare our $\mu_{\mathrm{g}}$ distribution with some currently available literature data. The results of the comparison are given in Table 6 and are shown in Fig. 21.

Two comments should be made concerning the content of Table 6 and Fig. 21. First, within some local zones the gas can be accumulated or exhausted by the radial gas flows, and this might be seen in observations, but it is not taken into account in our model. Second, the currently available literature $\mu_{\mathrm{g}}$ and $\mu_{\mathrm{s}}$ values are the subject of constant changes and 


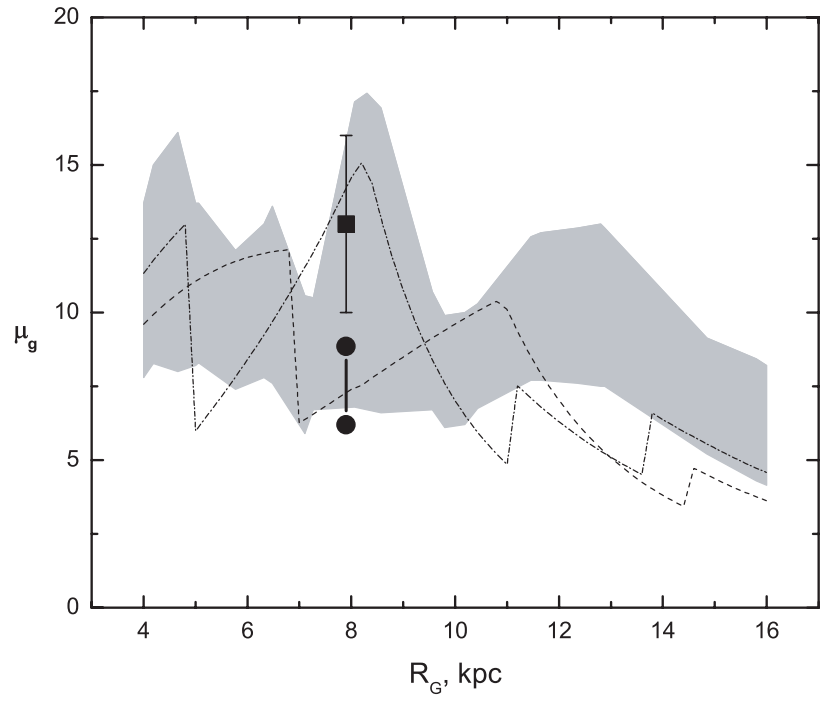

Fig. 21. Comparison between the calculated gas surface density (model L20 - dashed line and L27 - dash - dotted line) and semiempirical data from Olling \& Merrifiels (1998), Wolfire et al. (2003), and Dame (1993). The position of these data is indicated by the shaded area. The lower and upper boundaries correspond respectively to uncorrected and corrected for the helium content the gas surface density (only the corrected data were used from Dame 1993). The two connected filled circles are the estimates for the solar vicinity from Dickey (1993), the lower one is not corrected, and the upper one is corrected for the helium content; filled square - an estimate from Kuijken \& Gilmore (1991).

improvements, and it is important to know with the highest precision these values in order to constrain our models.

\section{Conclusions}

With newly analysed 12 galactic Cepheids situated within the range of galactocentric distances from 9 to $12 \mathrm{kpc}$ we have enlarged our homogeneous sample of stars used for galactic abundance gradient investigation to 122 objects. The results obtained in the present study in conjunction with those reported in Papers I-IV allow one to make the following conclusions:

1. The abundance distribution in galactic disc shows a multimodal structure. In particular, a wriggle-like structure of the metallicity distribution is seen between 10 and $11 \mathrm{kpc}$. This transition zone separates two regions where the abundance gradients are rather small. Another change in the slope in metallicity distribution is seen between 6 and $7 \mathrm{kpc}$.

2. Observed characteristics of the abundance distribution can be explained in the framework of a simple model of the disc chemical evolution supposing that spiral arms play an important role in the star formation process. Good agreement between our observed and modeled radial abundance distributions takes place under the following assumptions: - the star formation rate is a combination of two terms. One of them is proportional to the gas surface density, while the other also depends upon the relative velocity of the interstellar gas and spiral arms. The second term is negligibly small in the vicinity of a corotation resonance, it increases

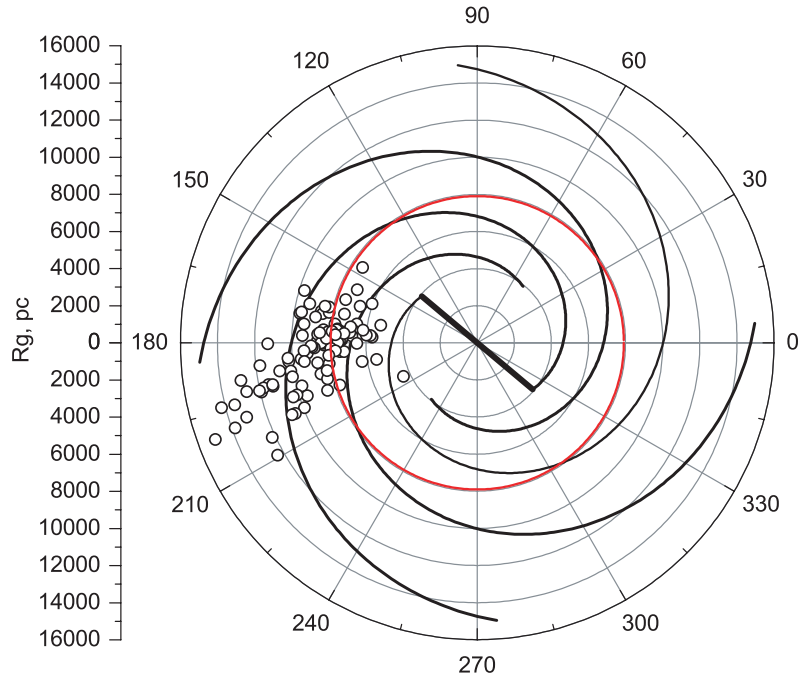

Fig. 22. Position of investigated Cepheids (Paper I-IV, and present study $)$ in galactocentric polar system. The solar circle $\left(R_{\mathrm{G}, \odot}=7.9 \mathrm{kpc}\right.$, red line), four logarithmic arms (pitch angle $p=13.8$, Ortiz \& Lépine 1993), and galactic bar (semi-magor axis of $3.9 \mathrm{kpc}$ and positional angle of $40^{\circ}$, López-Cerredoira et al. 2001) are schematically shown.

from the corotation towards the inner and outer Lindblad resonances, and equals zero inwards of the inner Lindblad resonance and outwards of the outer Lindblad resonance; - the coefficient of the star formation rate very slowly decreases with an increase of galactocentric distance; the characteristic time of infall rate is a function of galactocentric distance: it is smaller in the inner part of the Galaxy and increases towards the larger radii. The case of an exponential increase gives better results for the innermost part of the disc.

3. An agreement between observed radial abundance distribution of the iron and iron-group elements and calculated with the mentioned above assumptions seems to favour a spiral pattern speed $\Omega_{\mathrm{p}}=20 \mathrm{~km} \mathrm{~s}^{-1} \mathrm{kpc}^{-1}$.

One should note that some conclusions made above can possibly change if a larger sample of stars with $l$ ranging from, say, $60^{\circ}$ to $180^{\circ}$ is considered. In Fig. 22 we show our whole sample of investigated Cepheids superimposed on a simple sketch of a galactic disc with spiral arms. This sample begins in the vicinity of the galactic bar domain and conceivably stretches through several spiral arms in the narrow angular range. Can the radial elemental distributions be different for the different radial directions in the disc? To answer this question we have to significantly enlarge the number of investigated stars.

Acknowledgements. We are grateful to the CFHT Committee for allocation observing time to this project. We thank Yu. V . Beletsky for his help with a preliminary reduction of the spectra. The anonymous referee is kindly acknowledged for her/his very valuable comments and remarks. R.E.L and S.M.A acknowledge financial support from a Chretien International Research Grant (administered by the American Astronomical Society). 


\section{References}

Allen, C., \& Santillán, A. 1991, Rev. Mex. Astron. Astrophys., 22, 255

Amaral, L. H., \& Lépine, J. R. D. 1997, MNRAS, 286, 885

Andrievsky, S. M., Kovtyukh, V. V., Luck, R. E., et al. 2002a, A\&A, 381, 32 (Paper I)

Andrievsky, S. M., Bersier, D., Kovtyukh, V. V., et al. 2002b, A\&A, 384, 140 (Paper II)

Andrievsky, S. M., Kovtyukh, V. V., Luck, R. E., et al. 2002c, A\&A, 392, 491 (Paper III)

Avedisova, V. S. 1989, Astrophysics, 30, 83

Berdnikov, L. N., Ignatova, V. V., Pastukhova, E. N., \& Turner, D. G. 1997, AstL, 23, 177

Bersier, D., Burki, G., \& Burnet, M. 1994, A\&AS, 108, 9

Bissantz, N., Englmaier, P., \& Gerhard, O. 2003, MNRAS, 340, 949

Blitz, L., 1996, CO: Twenty-five years of millimeter-wave spectroscopy, ed. W. B., Latter, S. J. E., Radford, P. R., Jewell, J. G., Mangum, \& J., Bally (Tucson, Arizona: Kluwer Publishers), IAU Symp., 170, 11

Boissier, S., \& Prantzos, N. 1999, MNRAS, 307, 857

Byl, J., \& Ovenden, M. W. 1978, ApJ, 225, 496

Caputo, F., Marconi, M., Musella, I., \& Pont, F. 2001, A\&A, 372, 544

Chang, R.-X., Shu, Ch.-G., \& Hou, J.-L. 2002, ChJAA, 2, 226

Chiappini, C., Matteucci, F., \& Romano, D. 2001, ApJ, 554, 1044

Crézé, M., \& Mennessier, M. O. 1973, A\&A, 27, 281

Dame, T. M. 1993, Back to the Galaxy, ed. S. S. Holt, F. Verter (New York), AIP Conf. Proc., 278, 267

Dickey, J. M. 1993, The Minnesota lectures on clusters of galaxies and large-scale structure, ed. R. M. Humphreys (San Francisco), ASP Conf. Ser., 93

Englmaier, P., \& Gerhard, O. 1997, MNRAS, 287, 57

Fernández, D., Figueras, F., \& Torra, J. 2001, A\&A, 372, 833

Fernie, J. D., Evans, N. R., Beattie, B., \& Seager, S. 1995, IBVS, 4148,1

Friedli, D., Benz, W., \& Kennicutt, R. C. 1994, ApJ, 430, L105

Galazutdinov, G. A. 1992, Prepr. SAO RAS, 92

Gieren, W. P., Fouqué, P., \& Gomez, N. 1998, ApJ, 496, 17

Grevesse, N., Noels, A., \& Sauval, J. 1996, ASP Conf. Ser., 99, 117

Heyer, M. H., Dame T. M., \& Thaddeus P. 2000, The interstellar medium in M 31 and M 33, Proc. 232, WE-Heraeus-Seminar, ed. E. M. Berkhuijsen, R. Beck, \& R. A. M. Walterbos, Bad Honnef, Germany, 29

Hou, J. L., Prantzos, N., \& Boissier, S. 2000, A\&A, 362, 921

Kovtyukh, V. V., \& Andrievsky, S. M. 1999, A\&A, 351, 597

Kovtyukh, V. V., \& Gorlova, N. I. 2000, A\&A, 358, 587

Kulkarni, S. R., \& Heiles, C. 1987, Interstellar processes, ed. D. Hollenbach, \& H. Thronson (Dordrecht: Kluwer Publishers), 87

Lacey, C. G., \& Fall, S. M. 1985, ApJ, 290, 154

Laney, C. D., \& Stobie, R. S. 1993, MNRAS, 263, 921

Lépine, J. R. D., Mishurov, Yu. N., \& Dedikov, S. Yu. 2001, AJ, 546, 324

Lin, C. C., Yuan, C., \& Shu, F. H. 1969, ApJ, 155, 721
Lockman, J. F. 1988, The outer Galaxy, ed. L. Blitz, \& J. F. Lockman (New York: Springer), 79

López-Corredoira, M., Hammersley, P. L., Garzón, F., et al. 2001, A\&A, 373, 139

Luck, R. E., Kovtyukh, V. V., \& Andrievsky, S. M. 2001, A\&A, 373, 589

Luck, R. E., Gieren, W. P., Andrievsky, S. M., et al. 2003, A\&A, 401, 939 (Paper IV)

Maeder, A. 1992, A\&A, 552, 591

Marochnik, L. S., Mishurov, Yu. N., \& Suchkov, A. A. 1972, Ap\&SS, 206, 285

Martin, P., \& Roy, J.-R. 1995, ApJ, 445, 161

Matteucci, F. 2003, Carnegie Obs. Astrophys. Ser., Origin and evolution of the elements, ed. A. McWilliam, M. Rauch (Cambridge Univ. Press), 1

McNamara, D. H., Madsen, J. B., Barnes, J., \& Ericksen, B. F. 2000, PASP, 112, 202

Mera, D., Chabrier, G., \& Schaeffer, R. 1998a, A\&A, 330, 937

Mera, D., Chabrier, G. \& Schaeffer, R. 1998b, A\&A, 330, 953

Mishurov, Yu. N., Pavlovskaya, E. D., \& Suchkov, A. A. 1979, SvA, 23, 147

Mishurov, Yu. N., Zenina, I. A., Dambis, A. K., Mel'nik, A. M., \& Rastorguev, A. S. 1997, A\&A, 323, 775

Mishurov, Yu. N., Lépine, J. R. D., \& Acharova, I. A. 2002, ApJ, 571, 113

Moffet, T. J., \& Barnes, III T. G. 1985, ApJS, 58, 843

Olling, R. P., \& Merrifield, M. R. 1998, MNRAS, 297, 943

Oort, J. H. 1974, The formation and dynamics of Galaxies, ed. J. R. Shakeshaft, (Dordrecht: Reidel), IAU Symp., 58, 375

Ortiz, R., \& Lépine, J. R. D. 1993, A\&A, 279, 90

Pavlovskaya, E. D., \& Suchkov, A. A. 1980, SvA, 24, 164

Pichardo, B., Martos, M., Moreno, E., \& Espresate, J. 2003, ApJ, 582, 230

Pilyugin, L. S. 2003, A\&A, 397, 109

Portinari, L., \& Chiosi, C. 1999, A\&A, 350, 827

Portinari, L., \& Chiosi, C. 2000, A\&A, 355, 929

Roberts, W. W. Jr. 1970, The spiral structure of our Galaxy, ed. W. Becker, \& G. I. Kontopoulos, (Dordrecht: Reidel), Proc. IAU Symp., 38, 415

Roberts, W.W. Jr. 1972, ApJ, 173, 259

Roy, J.-R. 1996, Barred galaxies, ed. R. Buta, D. A. Crocker, \& B. G. Elmegreen, ASP Conf. Ser., 91, 63

Schmidt, E. G., Chab, J. R., \& Reiswig, D. E. 1995, AJ, 109, 1239

Szabados, L. 1991, Mitt. Stern. Ung. Akad. Wiss., 96

Thon, R., \& Meusinger, H. 1998, A\&A, 338, 413

Tinsley, B. 1980, Fundamentals of cosmic physics, 5, 287

Tsujimoto, T., Nomoto, K., Yoshii, Y., et al. 1995, MNRAS, 277, 945

Twarog, B. A., Ashman, K. M., \& Antony-Twarog, B. J. 1997, AJ, 114,2556

Vinko, J. 1991, Ap\&SS, 183, 17

Wolfire, M. G., McKee, C. F., \& Hollenbach, D. 2003, ApJ, 587, 278

Wong, T., Blitz, L. 2002, ApJ, 569, 157 